Article

\title{
Hypersurfaces of a Sasakian Manifold
}

\author{
Haila Alodan ${ }^{1}$, Sharief Deshmukh ${ }^{1}\left(\mathbb{D}\right.$, Nasser Bin Turki $^{1}{ }^{\mathbb{D}}$ and Gabriel-Eduard Vîlcu ${ }^{2, *(\mathbb{D})}$ \\ 1 Department of Mathematics, College of Science, King Saud University, P.O. Box-2455, \\ Riyadh 11451, Saudi Arabia; halodan1@ksu.edu.sa (H.A.); shariefd@ksu.edu.sa (S.D.); \\ nassert@ksu.edu.sa (N.B.T.) \\ 2 Department of Cybernetics, Economic Informatics, Finance and Accountancy, \\ Petroleum-Gas University of Ploieşti, Bd. Bucureşti 39, 100680 Ploieşti, Romania \\ * Correspondence: gvilcu@upg-ploiesti.ro; Tel.: +40-244-575-847
}

Received: 3 May 2020; Accepted: 25 May 2020; Published: 1 June 2020

check for updates

\begin{abstract}
We extend the study of orientable hypersurfaces in a Sasakian manifold initiated by Watanabe. The Reeb vector field $\xi$ of the Sasakian manifold induces a vector field $\xi^{T}$ on the hypersurface, namely the tangential component of $\xi$ to hypersurface, and it also gives a smooth function $\rho$ on the hypersurface, which is the projection of the Reeb vector field on the unit normal. First, we find volume estimates for a compact orientable hypersurface and then we use them to find an upper bound of the first nonzero eigenvalue of the Laplace operator on the hypersurface, showing that if the equality holds then the hypersurface is isometric to a certain sphere. Also, we use a bound on the energy of the vector field $\nabla \rho$ on a compact orientable hypersurface in a Sasakian manifold in order to find another geometric condition (in terms of mean curvature and integral curves of $\xi^{T}$ ) under which the hypersurface is isometric to a sphere. Finally, we study compact orientable hypersurfaces with constant mean curvature in a Sasakian manifold and find a sharp upper bound on the first nonzero eigenvalue of the Laplace operator on the hypersurface. In particular, we show that this upper bound is attained if and only if the hypersurface is isometric to a sphere, provided that the Ricci curvature of the hypersurface along $\nabla \rho$ has a certain lower bound.
\end{abstract}

Keywords: hypersurface; Sasakian manifold; Laplace operator; eigenvalue

MSC: 53C42; 53C25; 53B25

\section{Introduction}

It is well known that Sasakian manifolds are considered the odd dimensional analogue of Kählerian manifolds and therefore Sasakian space forms can be regarded as the counterpart of complex space forms [1]. One of the important branches of differential geometry is the submanifold theory and here some very challenging topics come from the geometry of submanifolds in real, complex and Sasakian space forms. In this setting, in many studies, a key role is played by the Gauss, Codazzi and Ricci Equations for submanifolds, as these take a manageable form. The differential geometry of hypersurfaces in a complex space form has been widely studied over the years (see, e.g., [2-11]), but though Sasakian manifolds are very important due to their elegant geometry (see the excellent monograph [12]) as well as their important applications in theoretical physics (see [13] and the references therein), not as many studies have been realized for hypersurfaces in a Sasakian ambient space. In this context, a well known result is that of Watanabe (cf. [14]), who used the Obata's differential Equation (cf. $[15,16])$ in order to prove that a complete and connected totally umbilical hypersurface of a $(2 n+1)$-dimensional Sasakian manifold of constant mean curvature $H$ is isometric with a sphere of radius $\frac{1}{\sqrt{1+H^{2}}}$ in the Euclidean space. This study of Watanabe is further 
carried out in [17], where the author found two much stronger theorems that give sufficient conditions for a hypersurface in a Sasakian manifold to be isometric to a sphere.

Given an orientable hypersurface $M$ of a $(2 n+1)$-dimensional Sasakian manifold $\bar{M}(\varphi, \xi, \eta, g)$ with unit normal vector field $N$ and shape operator $A$, then there are two vector fields $\xi^{T}$ and $\mathbf{t}$ naturally defined on the hypersurface $M$. More exactly, $\xi^{T}$ is the tangential component of the Reeb vector field $\xi$ to $M$, while $\mathbf{t}$ is defined by $\varphi(N)=-\mathbf{t}$. Recall that the study of real hypersurfaces of complex and Sasakian space forms become more convenient, owing to a simpler form of Gauss Equation for expression of curvature tensor field of hypersurface and also due to a handy form of Codazzi Equation, which is lacking in the study of hypersurfaces of a general Sasakian manifold.

In this paper, we show that focussing on the investigation of hypersurfaces in general Sasakian manifolds, this deficiency can be compensated by the power of the Reeb field. In Section 2, we derive basic formulae for an orientable hypersurface $M$ of a $(2 n+1)$-dimensional Sasakian manifold $\bar{M}(\varphi, \xi, \eta, g)$. In Section 3, we find an estimate of volume for a compact orientable hypersurface $M$ and use it together with an additional condition, namely the vector field $\xi^{T}$ is a principal direction, to find an upper bound for the first nonzero eigenvalue $\lambda_{1}$ of the Laplace operator on $M$. Also, we show that if the eigenvalue $\lambda_{1}$ attains this upper bound, then $M$ is isometric to a sphere (cf. Theorems 1 and 2). Moreover, in the same section, we find other special conditions on a compact orientable hypersurface $M$ that assure both constancy of the mean curvature $H$ and the isometry of $M$ with a certain sphere (cf. Theorem 3).

In Section 4, we use an upper bound for the energy of the gradient vector field $\nabla \rho$ and the condition that the mean curvature $H$ of the compact orientable hypersurface $M$ in a $(2 n+1)$-dimensional Sasakian manifold is constant along the integral curves of $\xi^{T}$ in order to show that in this case, $H$ is also a constant and $M$ is isometric with a sphere $\mathbf{S}^{2 n}(r)$ with radius $r=\frac{1}{\sqrt{1+H^{2}}}$ (cf. Theorem 4). Finally, in the last section of the paper, we study hypersurfaces of constant mean curvature $H$, also known as CMC-hypersurfaces, in a $(2 n+1)$-dimensional Sasakian manifold $\bar{M}(\varphi, \xi, \eta, g)$. We prove that on a compact orientable CMC-hypersurface $M$, if $\xi^{T}$ is a principal direction with constant principal curvature $\mu$, then necessarily $H=\mu$. We show that for a compact orientable CMC-hypersurface $M$ with $A \xi^{T}=\mu \xi^{T}$, the first nonzero eigenvalue $\lambda_{1}$ of the Laplace operator satisfies $\lambda_{1} \leq 2 n\left(1+H^{2}\right)$ and the equality case holds for a certain hypersurface if and only if $M$ is isometric to the sphere $\mathbf{S}^{2 n}\left(\frac{1}{\sqrt{1+H^{2}}}\right)$ (cf. Theorem 5).

\section{Preliminaries}

Let $\bar{M}(\varphi, \xi, \eta, g)$ be a $(2 n+1)$-dimensional Sasakian manifold and $\bar{\nabla}$ be the Riemannian connection on $\bar{M}$. Then we have (cf. [1,12]):

$$
\begin{gathered}
\varphi^{2}(X)=-X+\eta(X) \xi, \quad \varphi(\xi)=0, \quad \eta \circ \varphi=0, \quad g(X, \xi)=\eta(X), \\
g(\varphi(X), \varphi(Y))=g(X, Y)-\eta(X) \eta(Y), \\
(\bar{\nabla} \varphi)(X, Y)=g(X, Y) \xi-\eta(Y) X, \quad \bar{\nabla}_{X} \xi=-\varphi X,
\end{gathered}
$$

for all $X, Y \in \mathcal{X}(\bar{M})$, where $\mathcal{X}(\bar{M})$ is the Lie algebra of smooth vector fields on $\bar{M}$, while the covariant derivative $\bar{\nabla} \varphi$ of $\varphi$ is defined by

$$
(\bar{\nabla} \varphi)(X, Y)=\bar{\nabla}_{X} \varphi(Y)-\varphi\left(\bar{\nabla}_{X} Y\right) .
$$

We denote by $\bar{R}, \overline{R i c}, \bar{Q}$ the curvature tensor field, the Ricci tensor field and the Ricci operator of the Sasakian manifold $\bar{M}(\varphi, \xi, \eta, g)$. Then, for all $X, Y \in \mathcal{X}(\bar{M})$, we have (cf. [1]):

$$
\bar{R}(X, Y) \xi=\eta(Y) X-\eta(X) Y, \quad \bar{Q}(\xi)=2 n \xi,
$$


where Ricci operator $\bar{Q}$ is a symmetric operator related to Ricci tensor $\overline{\operatorname{Ric}}$ by [18]

$$
\overline{\operatorname{Ric}}(X, Y)=g(\bar{Q}(X), Y) .
$$

Recall that for all vector fields $X, Y$ orthogonal to $\xi$, we have

$$
\overline{\operatorname{Ric}}(\varphi(X), \varphi(Y))=\overline{\operatorname{Ric}}(X, Y) .
$$

Let $M$ be an orientable hypersurface of a $(2 n+1)$-dimensional Sasakian manifold $\bar{M}(\varphi, \xi, \eta, g)$ with unit normal vector field $N$ and shape operator $A$. Then we have the Gauss and Weingarten formulae (cf. [19])

$$
\bar{\nabla}_{U} V=\nabla_{U} V+g(A U, V) N, \quad \bar{\nabla}_{U} N=-A U, \quad U, V \in \mathcal{X}(M),
$$

where we denote by the same letter $g$ the induced metric on $M$ and by $\nabla$ the Riemannian connection on $M$. Clearly, $\mathcal{X}(M)$ is the Lie algebra of smooth vector fields on $M$.

Please note that owing to skew-symmetry of the operator $\varphi, \varphi(N)$ is orthogonal to $N$ and we get a smooth vector field $\mathbf{t} \in \mathcal{X}(M)$, defined by $\varphi(N)=-\mathbf{t}$. Let $\alpha$ be smooth 1 -form on hypersurface $M$ dual to $\mathbf{t}$, that is, $\alpha(U)=g(\mathbf{t}, U), U \in \mathcal{X}(M)$. Also, we define an operator $F: \mathcal{X}(M) \rightarrow \mathcal{X}(M)$ by $F(U)=[\varphi(U)]^{T}$ - the tangential component of $\varphi(U)$ to the hypersurface $M$. Then we have

$$
\varphi(U)=F(U)+\alpha(U) N, \quad U \in \mathcal{X}(M)
$$

and it is easy to see that $F$ is a skew-symmetric operator.

Now, define a smooth function $\rho$ on the hypersurface $M$ by $\rho=g(\xi, N)$. Then we have

$$
\xi=\xi^{T}+\rho N
$$

where $\xi^{T} \in \mathcal{X}(M)$ is the tangential component of the Reeb vector field $\xi$. We denote by $\beta$ the smooth 1-form on $M$ dual to $\xi^{T}$, i.e, $\beta(U)=g\left(\xi^{T}, U\right)$. Then using $\varphi(N)=-\mathbf{t}$ and Equations (1), (2), (7) and (8), it follows that

$$
\begin{gathered}
\|\mathbf{t}\|^{2}=\left\|\xi^{T}\right\|^{2}=1-\rho^{2}, \\
F(\mathfrak{t})=-\rho \xi^{T}, \quad F\left(\xi^{T}\right)=\rho \mathbf{t}, \\
F^{2}(U)=-U+\alpha(U) \mathbf{t}+\beta(U) \xi^{T},
\end{gathered}
$$

and

$$
g(F(U), F(V))=g(U, V)-\alpha(U) \alpha(V)-\beta(U) \beta(V), \quad U, V \in \mathcal{X}(M) .
$$

Also, using Equations (3), (6), (8) and $\varphi(N)=-\mathbf{t}$, we conclude

$$
\begin{gathered}
\nabla_{U} \mathbf{t}=\rho U+F(A U), \quad \nabla_{U} \xi^{T}=-F(U)+\rho A U, \quad \nabla \rho=-A \xi^{T}-\mathbf{t}, \\
(\nabla F)(U, V)=g(U, V) \xi^{T}-\beta(V) U+\alpha(V) A U-g(A U, V) \mathbf{t},
\end{gathered}
$$

where $\nabla \rho$ is the gradient of the function $\rho$ and the covariant derivative $\nabla F$ of $F$ is given by

$$
(\nabla F)(U, V)=\nabla_{U} F(V)-F\left(\nabla_{U} V\right),
$$

for $U, V \in \mathcal{X}(M)$. 
Please note that $F$ is skew-symmetric and $A$ symmetric, we have $\operatorname{tr}(F \circ A)=0$ and using first two equations in Equation (13), we conclude that

$$
\operatorname{divt}=2 n \rho, \quad \operatorname{div} \xi^{T}=2 n \rho H,
$$

where $H$ is the mean curvature of the hypersurface $M$ given by $2 n H=\operatorname{tr} A$. Thus, if $M$ is a compact hypersurface of a $(2 n+1)$-dimensional Sasakian manifold $\bar{M}(\varphi, \xi, \eta, g)$, Equation (15) leads to

$$
\int_{M} \rho=0, \quad \int_{M} \rho H=0 .
$$

If $\lambda_{1}$ is the first nonzero eigenvalue of the Laplace operator $\Delta$ acting on smooth functions on $M$, then using first Equation in (16) we get

$$
\int_{M}\|\nabla \rho\|^{2} \geq \lambda_{1} \int_{M} \rho^{2}
$$

Using Equation (6), we have

$$
\bar{R}(U, V) N=-(\nabla A)(U, V)+(\nabla A)(V, U), \quad U, V \in \mathcal{X}(M) .
$$

Moreover, the curvature tensor $R$ of the hypersurface $M$ is given by

$$
R(U, V) W=[\bar{R}(U, V) W]^{T}+g(A V, W) A U-g(A U, W) A V .
$$

Choosing a local orthonormal frame $\left\{e_{1}, \ldots, e_{2 n}\right\}$ on the hypersurface and using Equation (19), we get the following expression for the Ricci tensor Ric of the hypersurface $M$

$$
\operatorname{Ric}(U, V)=2 n H g(A U, V)-g(A U, A V)+\sum_{i=1}^{2 n} \bar{R}\left(e_{i}, U ; V, e_{i}\right)
$$

Observe that

$$
\sum_{i=1}^{2 n} \bar{R}\left(e_{i}, U ; V, e_{i}\right)=\overline{\operatorname{Ric}}(U, V)-\bar{R}(N, U ; V, N)
$$

and consequently, we conclude

$$
\operatorname{Ric}(U, V)=2 n H g(A U, V)-g(A U, A V)+\overline{\operatorname{Ric}}(U, V)-\bar{R}(N, U ; V, N) .
$$

Also, note that on an orientable hypersurface $M$ of a $(2 n+1)$-dimensional Sasakian manifold $\bar{M}(\varphi, \xi, \eta, g)$ there are two globally defined orthogonal vector fields $\xi^{T}, \mathbf{t}$ and they span a plane section of the tangent bundle of $M$. Thus, we have the sectional curvature $K\left(\xi^{T}, \mathbf{t}\right)$ given by

$$
K\left(\xi^{T}, \mathbf{t}\right)=\frac{R\left(\xi^{T}, \mathbf{t} ; \mathbf{t}, \xi^{T}\right)}{\left\|\xi^{T}\right\|^{2}\|\mathbf{t}\|^{2}} .
$$

Lemma 1. Let $M$ be a compact orientable hypersurface of a $(2 n+1)$-dimensional Sasakian manifold $\bar{M}(\varphi, \xi, \eta, g)$. Then

$$
\int_{M} g\left(A \xi^{T}, \xi^{T}\right)=2 n \int_{M} H \rho^{2}
$$


Proof. Observe that Equation $\varphi(N)=-\mathbf{t}$ and (1) imply that $g\left(\mathbf{t}, \xi^{T}\right)=0$. Thus, using Equation (13), we have

$$
\xi^{T}(\rho)=-g\left(A \xi^{T}, \xi^{T}\right) .
$$

Also, using Equation (15), we have

$$
\begin{aligned}
\operatorname{div}\left(\rho \xi^{T}\right) & =\xi^{T}(\rho)+\rho \operatorname{div} \xi^{T} \\
& =\xi^{T}(\rho)+2 n H \rho^{2} .
\end{aligned}
$$

Integrating this Equation and using Equation (22), we get the desired result.

Lemma 2. Let $M$ be a compact orientable hypersurface of a $(2 n+1)$-dimensional Sasakian manifold $\bar{M}(\varphi, \xi, \eta, g)$. Then

$$
\int_{M} g\left(A \xi^{T}, \mathbf{t}\right)=\int_{M}\left[(2 n+1) \rho^{2}-1\right] .
$$

Proof. Observe that Equation (13) gives

$$
\begin{aligned}
\mathbf{t}(\rho) & =-g\left(A \xi^{T}, \mathbf{t}\right)-\|\mathbf{t}\|^{2} \\
& =-g\left(A \xi^{T}, \mathbf{t}\right)-\left(1-\rho^{2}\right) .
\end{aligned}
$$

Using this in $\operatorname{div}(\rho \mathbf{t})=\mathbf{t}(\rho)+\rho \operatorname{divt}$ and equation (15), we get

$$
\operatorname{div}(\rho \mathbf{t})=-g\left(A \xi^{T}, \mathbf{t}\right)-\left(1-\rho^{2}\right)+2 n \rho^{2} .
$$

Integrating above Equation, we obtain the result.

\section{Volume and First Eigenvalue Estimates}

In this section, first we find the volume estimate for a compact hypersurface $M$ of a $(2 n+1)$-dimensional Sasakian manifold $\bar{M}(\varphi, \xi, \eta, g)$ and use it to find an upper bound for the first nonzero eigenvalue $\lambda_{1}$ of the Laplace operator $\Delta$ acting on smooth functions of hypersurface $M$ under the assumption that $\xi^{T}$ is a principal direction.

Theorem 1. The volume $V(M)$ of a compact orientable hypersurface $M$ of a $(2 n+1)$-dimensional Sasakian manifold $\bar{M}(\varphi, \xi, \eta, g)$ satisfies

$$
\boldsymbol{V}(M) \leq \int_{M}\left[\left(4 n+1-\lambda_{1}\right) \rho^{2}+\left\|A \xi^{T}\right\|^{2}\right],
$$

where $\lambda_{1}$ is the first nonzero eigenvalue of the Laplace operator on $M$.

Proof. Using Equation (9) and (13), we have

$$
\begin{aligned}
\|\nabla \rho\|^{2} & =\left\|A \xi^{T}\right\|^{2}+\|\mathbf{t}\|^{2}+2 g\left(A \xi^{T}, \mathbf{t}\right) \\
& =\left\|A \xi^{T}\right\|^{2}+\left(1-\rho^{2}\right)+2 g\left(A \xi^{T}, \mathbf{t}\right) .
\end{aligned}
$$

Integrating above Equation and using Lemma 2, we get

$$
\int_{M}\|\nabla \rho\|^{2}=\int_{M}\left[-1+(4 n+1) \rho^{2}+\left\|A \xi^{T}\right\|^{2}\right] .
$$


Using inequality (17), we derive

$$
\lambda_{1} \int_{M} \rho^{2} \leq \int_{M}\left[-1+(4 n+1) \rho^{2}+\left\|A \xi^{T}\right\|^{2}\right]
$$

and this gives the required estimate.

Now, suppose that the vector field $\xi^{T}$ is a principal direction of the hypersurface $M$ with constant principal curvature $\mu$, that is, $A \xi^{T}=\mu \xi^{T}$. Then it turns out that $\mathbf{t}$ is also a principal direction as seen in the following.

Lemma 3. If $M$ is a connected orientable hypersurface of a $(2 n+1)$-dimensional Sasakian manifold $\bar{M}(\varphi, \xi, \eta, g)$ and $A \xi^{T}=\mu \xi^{T}$ for a constant $\mu$, then $A \mathbf{t}=\mu \mathbf{t}$.

Proof. Suppose $A \xi^{T}=\mu \xi^{T}$ holds for a constant $\mu$. Then the last Equation in (13), gives $\nabla \rho=-\mu \xi^{T}-\mathbf{t}$, and using first two Equations in (13), we derive the following expression for the Hessian operator $A_{\rho}$ of the function $\rho$

$$
A_{\rho}(U)=-\mu[-F(U)+\rho A U]-[\rho U+F(A U)]
$$

that is,

$$
A_{\rho}(U)=-\rho(\mu A U+U)+\mu F(U)-F(A U), \quad V \in \mathcal{X}(M) .
$$

Note the Hessian operator $A_{\rho}$ is symmetric and thus, using above equation, we conclude that

$$
2 \mu g(F(U), V)=g(F(A U)+A F(U), V), \quad U, V \in \mathcal{X}(M),
$$

that is,

$$
2 \mu F(U)=F(A U)+A F(U) \quad U, V \in \mathcal{X}(M) .
$$

Taking $U=\xi^{T}$ in above equation and using Equation (10), we get

$$
2 \mu \rho \mathbf{t}=\mu \rho \mathbf{t}+\rho A \mathbf{t}
$$

that is

$$
\rho(A \mathbf{t}-\mu \mathbf{t})=0
$$

If $\rho=0$, then Equation (9) implies that both $\xi^{T}, \mathbf{t}$ are unit vector fields and as $\varphi(N)=-\mathbf{t}$, we get $g\left(\xi^{T}, \mathbf{t}\right)=-g(\xi, \varphi(N))=0$. Also, as $\rho=0$, Equation (13) implies $\mu \xi^{T}=-\mathbf{t}$ and taking the inner product with $\mathbf{t}$ gives $\|\mathbf{t}\|^{2}=0$ a contradiction to the fact that $\mathbf{t}$ is a unit vector field (under the assumption $\rho=0$ ). Hence $\rho \neq 0$ on $M$. Whereas $M$ is connected and $\rho \neq 0$, Equation (25) implies $A \mathbf{t}=\mu \mathbf{t}$.

Now, we shall prove the main results of this section.

Theorem 2. Let $M$ be a compact and connected orientable hypersurface of $a(2 n+1)$-dimensional Sasakian manifold $\bar{M}(\varphi, \xi, \eta, g)$. If $A \xi^{T}=\mu \xi^{T}$ for a constant $\mu$ and the squared length of shape operator is bounded above by $2 n c$ for a constant $c, 0<c \leq 1$, then the first nonzero eigenvalue $\lambda_{1}$ of Laplace operator on $M$ satisfies

$$
\lambda_{1} \leq 4 n+1-c
$$

Moreover, if equality holds, then $M$ is isometric to the sphere $\mathbf{S}^{2 n}\left(\frac{1}{\sqrt{1+\mu^{2}}}\right)$. 
Proof. Suppose $M$ is a compact and connected orientable hypersurface satisfying $A \xi^{T}=\mu \xi^{T}$ for a constant $\mu$ and $\|A\|^{2} \leq 2 n c$, for a constant $c, 0<c \leq 1$. Then by Lemma 3, we have $A \mathbf{t}=\mu \mathbf{t}$. Now, define operators $\Gamma$ and $\Psi$ by

$$
\Gamma=F \circ A+A \circ F, \quad \Psi=F \circ A-A \circ F .
$$

Then it follows that $\Gamma$ is skew-symmetric and $\Psi$ is symmetric with $\operatorname{tr} \Psi=0$ and we have

$$
F(A U)=\frac{1}{2}[\Gamma(U)+\Psi(U)], \quad U \in \mathcal{X}(M),
$$

and

$$
\|F \circ A\|^{2}=\frac{1}{4}\|\Gamma\|^{2}+\frac{1}{4}\|\Psi\|^{2},
$$

where we have used $\operatorname{tr}(\Gamma \circ \Psi)=0$. Using Equation (24) (which holds for $A \xi^{T}=\mu \xi^{T}$ ), we conclude

$$
2 \mu F=\Gamma .
$$

Please note that for a local orthonormal frame $\left\{e_{1}, \ldots, e_{2 n}\right\}$ on $M$, using Equation (12) we have

$$
\begin{aligned}
\|F\|^{2} & =\sum_{i=1}^{2 n} g\left(F\left(e_{i}\right), F\left(e_{i}\right)\right) \\
& =2 n-\left\|\xi^{T}\right\|^{2}-\|\mathbf{t}\|^{2} \\
& =2 n-2\left(1-\rho^{2}\right),
\end{aligned}
$$

where we have used Equation (9). Thus, the above Equation and (27) give

$$
\frac{1}{4}\|\Gamma\|^{2}=2 \mu^{2}\left(n-1+\rho^{2}\right) .
$$

Also, using Equation (12) we have

$$
\begin{aligned}
\|F \circ A\|^{2} & =\sum_{i=1}^{2 n} g\left(F\left(A e_{i}\right), F\left(A e_{i}\right)\right)=\|A\|^{2}-\left\|A \xi^{T}\right\|^{2}-\|A \mathbf{t}\|^{2} \\
& =\|A\|^{2}-2 \mu^{2}\left(1-\rho^{2}\right) .
\end{aligned}
$$

Combining Equations (26), (28) and (29), we conclude

$$
\|A\|^{2}=2 n \mu^{2}+\frac{1}{4}\|\Psi\|^{2},
$$

that is,

$$
\mu^{2}=\frac{1}{2 n}\left(\|A\|^{2}-\frac{1}{4}\|\Psi\|^{2}\right) .
$$

On the other hand, Theorem 1 in our setting implies

$$
V(M) \leq \int_{M}\left[\left(4 n+1-\lambda_{1}\right) \rho^{2}+\mu^{2}\left(1-\rho^{2}\right)\right] .
$$

Thus, using Equation (30) in above inequality, we conclude

$$
\boldsymbol{V}(M) \leq \int_{M}\left[\left(4 n+1-\lambda_{1}\right) \rho^{2}+\frac{\left(1-\rho^{2}\right)}{2 n}\left(\|A\|^{2}-\frac{1}{4}\|\Psi\|^{2}\right)\right] .
$$


Using next $\|A\|^{2} \leq 2 n c$, we get

$$
V(M) \leq \int_{M}\left[\left(4 n+1-\lambda_{1}-c\right) \rho^{2}+c-\frac{\left(1-\rho^{2}\right)}{8 n}\|\Psi\|^{2}\right],
$$

that is,

$$
(1-c) \boldsymbol{V}(M) \leq \int_{M}\left(4 n+1-\lambda_{1}-c\right) \rho^{2}-\int_{M} \frac{\left(1-\rho^{2}\right)}{8 n}\|\Psi\|^{2} .
$$

However, the above inequality implies

$$
(1-c) \boldsymbol{V}(M) \leq\left(4 n+1-\lambda_{1}-c\right) \int_{M} \rho^{2}
$$

and as $1-c \geq 0$, we conclude $\left(4 n+1-\lambda_{1}-c\right) \geq 0$, that proves $\lambda_{1} \leq 4 n+1-c$.

If the equality $\lambda_{1}=4 n+1-c$ holds, then inequality (31) implies $c=1$ and $\left(1-\rho^{2}\right)\|\Psi\|^{2}=0$. If $\left(1-\rho^{2}\right)=0$, then $\rho= \pm 1$ together with first equation in (16) gives a contradiction. Hence on connected $M$, we get $\Psi=0$, i.e., $F \circ A=A \circ F$, and consequently $\Gamma=2 F \circ A$. Then Equation (27), gives $\mu F(U)=F(A U)$, i.e., $F(A U-\mu U)=0$. Operating $F$ in this last equation and using (11), we conclude

$$
A U-\mu U=\alpha(A U-\mu U) \mathbf{t}+\beta(A U-\mu U) \xi^{T}, \quad U \in \mathcal{X}(M) .
$$

On the other hand, it is easy to see that $A \mathbf{t}=\mu \mathbf{t}$ implies

$$
\alpha(A U-\mu U)=g(A U-\mu U, \mathbf{t})=\mu g(U, \mathbf{t})-\mu g(U, \mathbf{t})=0 .
$$

Similarly, we obtain $\beta(A U-\mu U)=0$ and consequently Equation (32), gives $A U=\mu U$, $U \in \mathcal{X}(M)$. Hence, $M$ is totally umbilical hypersurface of the Sasakian manifold $\bar{M}(\varphi, \xi, \eta, g)$ with constant mean curvature $\mu$ and therefore isometric to the sphere $\mathbf{S}^{2 n}\left(\frac{1}{\sqrt{1+\mu^{2}}}\right)$ (cf. [14]).

In the next result, we use a bound on the squared length of the operator $\Psi$ to find conditions on a complete and connected hypersurface of a Sasakian manifold to be isometric to a certain sphere.

Theorem 3. Let $M$ be a complete and connected orientable hypersurface of a $(2 n+1)$-dimensional Sasakian manifold $\bar{M}(\varphi, \xi, \eta, g)$ with mean curvature $H$. If $A \xi^{T}=\mu \xi^{T}$ for a constant $\mu$ and the squared length of operator $\Psi$ satisfies

$$
\|\Psi\|^{2} \leq 8\left(H^{2}-\mu^{2}\right)
$$

then $H$ is a constant and $M$ is isometric to the sphere $\mathbf{S}^{2 n}\left(\frac{1}{\sqrt{1+H^{2}}}\right)$.

Proof. Since, $A \xi^{T}=\mu \xi^{T}$, by Lemma 3, we have $A \mathbf{t}=\mu \mathbf{t}$. Now, using Equation (30), we have

$$
\|A\|^{2}=2 n \mu^{2}+\frac{1}{4}\|\Psi\|^{2},
$$

that is,

$$
\|A\|^{2}-2 n H^{2}=\frac{1}{4}\|\Psi\|^{2}-2 n\left(H^{2}-\mu^{2}\right) .
$$

Now, using the bound on $\|\Psi\|^{2}$, we conclude $\|A\|^{2}-2 n H^{2} \leq 0$. However, Schwartz's inequality implies $\|A\|^{2}-2 n H^{2} \geq 0$ and thus we have the equality $\|A\|^{2}=2 n H^{2}$, which holds if and only if

$$
A=H I \text {, }
$$


where I stands for the identity operator. Please note that in view of Equation (33), we have $A \xi^{T}=H \xi^{T}$, and the hypothesis $A \xi^{T}=\mu \xi^{T}$, we conclude

$$
(H-\mu) \xi^{T}=0 .
$$

If $\xi^{T}=0$, then by Equation (9), we get $\rho^{2}=1$ and consequently, $\mathbf{t}=0$. In this case, Equation (11) implies $F^{2}=-I$. Now, using the second Equations in (13) and (33), we have $F(U)=\rho H U$. Combining the above relation with $F^{2}=-I$ we get $H^{2}=-1$, hence a contradiction. Therefore, as $M$ is connected, Equation (34) implies $H=\mu$, that is, $H$ is a constant. Thus, by Equation (33), we see that $M$ is a totally umbilical hypersurface of constant mean curvature $H$ and consequently it is isometric to the sphere $\mathbf{S}^{2 n}\left(\frac{1}{\sqrt{1+H^{2}}}\right)$.

\section{A Bound on Energy of a Vector Field}

Recall that on a compact Riemannian manifold $(M, g)$, the energy of a smooth vector field $\mathbf{u}$ on $M$ is defined by

$$
E(\mathbf{u})=\frac{1}{2} \int_{M}\|\mathbf{u}\|^{2}
$$

In this section, we use a bound on the energy of the vector field $\nabla \rho$ on a compact orientable hypersurface $M$ to find another condition under which $M$ is isometric to a sphere.

Theorem 4. Let $M$ be a compact and connected orientable hypersurface of a $(2 n+1)$-dimensional Sasakian manifold $\bar{M}(\varphi, \xi, \eta, g)$ with unit normal $N$, mean curvature $H$ and sectional curvature $K\left(\xi^{T}, \mathbf{t}\right)>0$. If the energy of $\nabla \rho$ satisfies

$$
E(\nabla \rho) \leq \int_{M}\left[n H^{2}+\frac{1}{2} \overline{\operatorname{Ric}}(N, N)\right] \rho^{2}
$$

and $H$ is constant along the integral curves of $\xi^{T}$, then $H$ is a constant and $M$ is isometric to the sphere $\mathbf{S}^{2 n}\left(\frac{1}{\sqrt{1+H^{2}}}\right)$.

Proof. As the mean curvature $H$ is constant along the integral curves of $\xi^{T}$, we have

$$
\sum_{i=1}^{2 n} g\left((\nabla A)\left(\xi^{T}, e_{i}\right), e_{i}\right)=0
$$

where $\left\{e_{1}, \ldots, e_{2 n}\right\}$ is a local orthonormal frame on $M$. Now, using both Equations (18) and (35), we get

$$
\sum_{i=1}^{2 n} g\left((\nabla A)\left(e_{i}, \xi^{T}\right), e_{i}\right)+\sum_{i=1}^{2 n} g\left(\bar{R}\left(e_{i}, \xi^{T}\right) N, e_{i}\right)=0 .
$$

As $\left\{e_{1}, \ldots, e_{2 n}, N\right\}$ is local orthonormal frame on $\bar{M}$ and $A$ is a symmetric operator, above equation takes the form

$$
g\left(\xi^{T}, \sum_{i=1}^{2 n}(\nabla A)\left(e_{i}, e_{i}\right)\right)+\overline{\operatorname{Ric}}\left(\xi^{T}, N\right)=0 .
$$

However, taking into account (4), we derive

$$
\begin{aligned}
\overline{\operatorname{Ric}}\left(\xi^{T}, N\right) & =\overline{\operatorname{Ric}}(\xi-\rho N, N) \\
& =\overline{\operatorname{Ric}}(\xi, N)-\rho \overline{\operatorname{Ric}}(N, N) \\
& =2 n \rho-\rho \overline{\operatorname{Ric}}(N, N) .
\end{aligned}
$$


Thus, Equation (36) takes the form

$$
g\left(\xi^{T}, \sum_{i=1}^{2 n}(\nabla A)\left(e_{i}, e_{i}\right)\right)+2 n \rho-\rho \overline{\operatorname{Ric}}(N, N)=0 .
$$

We use now the second equation in (13), to compute

$$
\operatorname{div}\left(A \xi^{T}\right)=\sum_{i=1}^{2 n} g\left(-F\left(e_{i}\right)+\rho A e_{i}, A e_{i}\right)+g\left(\xi^{T}, \sum_{i=1}^{2 n}(\nabla A)\left(e_{i}, e_{i}\right)\right) .
$$

Using $\operatorname{tr}(F \circ A)=0$ in above Equation, we conclude

$$
\operatorname{div}\left(A \xi^{T}\right)=\rho\|A\|^{2}+g\left(\xi^{T}, \sum_{i=1}^{2 n}(\nabla A)\left(e_{i}, e_{i}\right)\right),
$$

which in view of Equation (37) implies

$$
\operatorname{div}\left(A \xi^{T}\right)=\rho\|A\|^{2}+\rho \overline{\operatorname{Ric}}(N, N)-2 n \rho .
$$

Now, using Equation (13) in (38), we get

$$
\operatorname{div}\left(\rho A \xi^{T}\right)=-\left\|A \xi^{T}\right\|^{2}-g\left(\mathbf{t}, A \xi^{T}\right)+\rho^{2}\|A\|^{2}+\rho^{2} \overline{\operatorname{Ric}}(N, N)-2 n \rho^{2} .
$$

Integrating above equation and using Lemma 2, we conclude

$$
\int_{M}\left[-\left\|A \xi^{T}\right\|^{2}+1-(4 n+1) \rho^{2}+\rho^{2}\|A\|^{2}+\rho^{2} \overline{\operatorname{Ric}}(N, N)\right]=0 .
$$

Now, using again (13), we obtain

$$
\|\nabla \rho\|^{2}=\left\|A \xi^{T}\right\|^{2}+\|\mathbf{t}\|^{2}+2 g\left(\mathbf{t}, A \xi^{T}\right) .
$$

Integrating above equation and using $\|\mathbf{t}\|^{2}=1-\rho^{2}$ and Lemma 2, we get

$$
\int_{M}-\left\|A \xi^{T}\right\|^{2}=\int_{M}\left[(4 n+1) \rho^{2}-1-\|\nabla \rho\|^{2}\right] .
$$

Inserting next the above Equation in (39), we get

$$
\begin{aligned}
\int_{M} \rho^{2}\|A\|^{2} & =\int_{M}\left[\|\nabla \rho\|^{2}-\rho^{2} \overline{\operatorname{Ric}}(N, N)\right] \\
& =2 E(\nabla \rho)-\int_{M} \rho^{2} \overline{\operatorname{Ric}}(N, N),
\end{aligned}
$$

that is,

$$
\int_{M} \rho^{2}\left(\|A\|^{2}-2 n H^{2}\right)=2 E(\nabla \rho)-\int_{M} \rho^{2}\left[2 n H^{2}+\overline{\operatorname{Ric}}(N, N)\right] .
$$

If the energy $E(\nabla \rho)$ satisfies the given condition in hypothesis, then Equation (40) reads

$$
\int_{M} \rho^{2}\left(\|A\|^{2}-2 n H^{2}\right) \leq 0 .
$$


Using Schwartz's inequality $\|A\|^{2} \geq 2 n H^{2}$ in inequality (41), we conclude

$$
\rho^{2}\left(\|A\|^{2}-2 n H^{2}\right)=0 .
$$

If $\rho=0$, then $\left\{\xi^{T}, \mathbf{t}\right\}$ is an orthonormal set globally defined on $M$ and equations in (13) take the form

$$
\nabla_{U} \xi^{T}=-F(U), \quad \nabla_{U} \mathbf{t}=F(A U), \quad A \xi^{T}=-\mathbf{t} .
$$

Also, the two equations of (10) imply $F(\mathbf{t})=0$ and $F\left(\xi^{T}\right)=0$ and thus using Equation (43), we get

$$
\nabla_{\mathbf{t}} \xi^{T}=0, \nabla_{\xi^{T}} \mathbf{t}=0, \nabla_{\mathbf{t}} \mathbf{t}=F(A \mathbf{t}) .
$$

Thus, we compute

$$
R\left(\xi^{T}, \mathbf{t}\right) \mathbf{t}=\nabla_{\xi^{T}} F(A \mathbf{t})
$$

and consequently,

$$
\begin{aligned}
K\left(\xi^{T}, \mathbf{t}\right) & =R\left(\xi^{T}, \mathbf{t} ; \mathbf{t}, \xi^{T}\right) \\
& =g\left(\nabla_{\xi^{T}} F(A \mathbf{t}), \xi^{T}\right) \\
& =\xi^{T} g\left(F(A \mathbf{t}), \xi^{T}\right)-g\left(F(A \mathbf{t}), \nabla_{\xi^{T} \xi^{T}}\right) \\
& =0,
\end{aligned}
$$

which is contrary to the hypothesis. Hence, $\rho \neq 0$ and due to the fact that $M$ is connected, it follows that Equation (42) implies $\|A\|^{2}=2 n H^{2}$ and this inequality holds if and only if

$$
A=H I .
$$

Now, we proceed to show that $H$ is a constant. In view of Equation (44), the equations in (13) change to

$$
\nabla_{U} \mathbf{t}=\rho U+H F(U), \quad \nabla_{U} \xi^{T}=-F(U)+\rho H U, \quad \nabla \rho=-H \xi^{T}-\mathbf{t} .
$$

Using the above Equations, we compute the Hessian operator $A_{\rho}(U)=\nabla_{U} \nabla \rho$ and get the following

$$
A_{\rho}(U)=-\left(1+H^{2}\right) \rho U-U(H) \xi^{T} .
$$

Please note that $A_{\rho}$ is symmetric, and as such the above equation gives

$$
U(H) g\left(\xi^{T}, V\right)=V(H) g\left(\xi^{T}, U\right), \quad U, V \in \mathcal{X}(M) .
$$

Choosing $V=\xi^{T}$ in the above equation and using hypothesis that $H$ is constant along the integral curves of $\xi^{T}$, we get

$$
U(H)\left\|\xi^{T}\right\|^{2}=0 .
$$

If $\left\|\xi^{T}\right\|^{2}=0$, we derive $\rho= \pm 1$, which gives a contradiction to the integral formula (16). Hence, as $M$ is connected, Equation (45) implies $U(H)=0, U \in \mathcal{X}(M)$. Therefore, $H$ is a constant and by Equation (44) we get that $M$ is a totally umbilical hypersurface of constant mean curvature $H$. Consequently, we deduce that $M$ is isometric to $\mathbf{S}^{2 n}\left(\frac{1}{\sqrt{1+H^{2}}}\right)$.

Recall that the odd dimensional unit sphere $\mathbf{S}^{2 n+1}$ viewed as a hypersurface of the complex space $\mathbf{C}^{n+1}$ admits a standard Sasakian structure $(\varphi, \xi, \eta, g)$ (see [1] for details). As a particular case of the above theorem, we have the following result. 
Corollary 1. Let $M$ be a compact and connected orientable hypersurface of the unit sphere $\mathbf{S}^{2 n+1}$ with unit normal $N$, mean curvature $H$ and sectional curvature $K\left(\xi^{T}, \mathbf{t}\right)>0$. If the energy of $\nabla \rho$ satisfies

$$
E(\nabla \rho) \leq n \int_{M}\left(1+H^{2}\right) \rho^{2}
$$

and $H$ is constant along the integral curves of $\xi^{T}$, then $H$ is a constant and $M$ is isometric to the sphere $\mathbf{S}^{2 n}\left(\frac{1}{\sqrt{1+H^{2}}}\right)$. Moreover, the converse holds.

Proof. For the unit sphere $\mathbf{S}^{2 n+1}$ with unit normal $N$, we have $\overline{\operatorname{Ric}}(N, N)=2 n$ and the bound on $E(\nabla \rho)$ in the Theorem 4 becomes as in this statement. Therefore, $M$ is isometric to the sphere $\mathbf{S}^{2 n}\left(\frac{1}{\sqrt{1+H^{2}}}\right)$. Conversely, if $M$ is isometric to the small sphere $\mathbf{S}^{2 n}\left(\frac{1}{\sqrt{1+H^{2}}}\right)$, then it is totally umbilical hypersurface of constant mean curvature $H$, i.e., $A=H I$. In this situation, Equation (13) implies $\nabla \rho=$ $-H \xi^{T}-\mathbf{t}$. Thus, using Equation (15), we infer $\Delta \rho=-2 n\left(1+H^{2}\right) \rho$, i.e., $\rho \Delta \rho=-2 n\left(1+H^{2}\right) \rho^{2}$. Integrating this equation by parts, we get

$$
E(\nabla \rho)=\frac{1}{2} \int_{M}\|\nabla \rho\|^{2}=n \int_{M}\left(1+H^{2}\right) \rho^{2} .
$$

Hence, all the conditions in the statement are met.

\section{CMC-Hypersurfaces}

In this section, we study compact and connected oriented hypersurfaces of constant mean curvature (briefly CMC-hypersurfaces) of a Sasakian manifold. It is interesting to note that on compact orientable CMC hypersurfaces, if $\xi^{T}$ is a principal direction, then $A \xi^{T}=H \xi^{t}$ holds, where $H$ is the constant mean curvature. We also find a sharp upper bound for the first nonzero eigenvalue of the Laplace operator on compact and orientable CMC-hypersurfaces with $\xi^{T}$ a principal direction.

Lemma 4. Let $M$ be compact and connected orientable hypersurface of constant mean curvature $H$ of a $(2 n+$ 1)-dimensional Sasakian manifold $\bar{M}(\varphi, \xi, \eta, g)$. If $A \xi^{t}=\mu \xi^{T}$ for a constant $\mu$, then $H=\mu$ holds.

Proof. Using Lemma 1 and Lemma 2, we have

$$
\mu \int_{M}\left(1-\rho^{2}\right)=2 n H \int_{M} \rho^{2}
$$

and

$$
\int_{M}\left[(2 n+1) \rho^{2}-1\right]=0 .
$$

These two equations imply

$$
\int_{M}(2 n+1) \mu \rho^{2}=(2 n H+\mu) \int_{M} \rho^{2},
$$

that is,

$$
2 n(H-\mu) \int_{M} \rho^{2}=0 .
$$

If $\rho=0$, then Equation (9) implies that the set $\left\{\xi^{T}, \mathbf{t}\right\}$ is an orthonormal set. However, the last equation in (13) and $A \xi^{t}=\mu \xi^{T}$, gives $\mu \xi^{T}=-\mathbf{t}$. Taking the inner product in this last equation, gives $\|\mathbf{t}\|^{2}=0$ a contradiction. Hence, $\rho \neq 0$ and consequently, Equation (46) confirms that $H=\mu$. 
Theorem 5. Let $M$ be compact and connected orientable hypersurface of constant mean curvature $H$ of $a(2 n+1)$-dimensional Sasakian manifold $\bar{M}(\varphi, \xi, \eta, g)$ and $A \xi^{t}=\mu \xi^{T}$ for a constant $\mu$. Then the first nonzero eigenvalue $\lambda_{1}$ of the Laplace operator on $M$ satisfies

$$
\lambda_{1} \leq 2 n\left(1+H^{2}\right) .
$$

Moreover, the equality holds if and only if $M$ is isometric to the sphere $\mathbf{S}^{2 n}\left(\frac{1}{\sqrt{1+H^{2}}}\right)$, provided that the Ricci curvature of the hypersurface satisfies

$$
\operatorname{Ric}(\nabla \rho, \nabla \rho) \geq(2 n-1)\left(1+H^{2}\right)\|\nabla \rho\|^{2} .
$$

Proof. By Lemma 4, we have $H=\mu$ and then Theorem 1 implies

$$
\boldsymbol{V}(M) \leq \int_{M}\left[\left(4 n+1-\lambda_{1}\right) \rho^{2}+H^{2}\left(1-\rho^{2}\right)\right]
$$

that is

$$
\left(1-H^{2}\right) \boldsymbol{V}(M) \leq\left(4 n+1-H^{2}-\lambda_{1}\right) \int_{M} \rho^{2} .
$$

Also, as $A \xi^{t}=\mu \xi^{T}$, we have $g\left(A \xi^{t}, \mathbf{t}\right)=0$ and Lemma 2 implies

$$
V(M)=(2 n+1) \int_{M} \rho^{2} .
$$

Inserting this equation in inequality (48), we conclude

$$
\left[\lambda_{1}-2 n\left(1+H^{2}\right)\right] \int_{M} \rho^{2} \leq 0 .
$$

Now, using the argument given in the proof of Lemma 4 , we see that $\rho \neq 0$. Hence, from above inequality, we conclude $\lambda_{1} \leq 2 n\left(1+H^{2}\right)$.

Suppose now the equality $\lambda_{1}=2 n\left(1+H^{2}\right)$ is valid and that inequality (47) holds. Using $A \xi^{t}=\mu \xi^{T}$ and $\mu=H$ in last Equation of (13), we have

$$
\nabla \rho=-H \xi^{T}-\mathbf{t}
$$

Using Equations (15) and (49) and $\Delta \rho=\operatorname{div}(\nabla \rho)$, we get

$$
\Delta \rho=-2 n\left(1+H^{2}\right) \rho=-\lambda_{1} \rho .
$$

Also, using (13) and (49), we can compute the Hessian operator $A_{\rho}$ as

$$
A_{\rho}(U)=-H(-F(U)+\rho A U)-(\rho U+F(A U))
$$

or

$$
A_{\rho}(U)=-\rho(H A+I)(U)+F(H I-A)(U), \quad U \in \mathcal{X}(M) .
$$

Please note that for a local orthonormal frame $\left\{e_{1}, \ldots, e_{2 n}\right\}$ on $M$, owing to symmetry of operator $(H A+I)$ and skew-symmetry of operator $F(H I-A)$, we have

$$
\sum_{i=1}^{2 n} g\left((H A+I)\left(e_{i}\right), F(H I-A)\left(e_{i}\right)\right)=0 .
$$


Thus, Equation (51) gives

$$
\left\|A_{\rho}\right\|^{2}=\rho^{2}\|H A+I\|^{2}+\|F(H I-A)\|^{2} .
$$

On the other hand, it is clear that Equation (2) implies $\|F(U)\|^{2}=\|U\|^{2}-\alpha(U)^{2}-\beta(U)^{2}$. Observe that

$$
\begin{aligned}
\sum_{i=1}^{2 n} \alpha\left(H e_{i}-A e_{i}\right)^{2} & =\sum_{i=1}^{2 n} g\left(H e_{i}-A e_{i}, \mathbf{t}\right)^{2} \\
& =H^{2}\|\mathbf{t}\|^{2}-2 H g(\mathbf{t}, A \mathbf{t})+\|A \mathbf{t}\|^{2}
\end{aligned}
$$

We know that $A \xi^{t}=\mu \xi^{T}$ implies $A \mathbf{t}=\mu \mathbf{t}($ cf. Lemma 3 ) and that $H=\mu$. Consequently, the above equation gives

$$
\sum_{i=1}^{2 n} \alpha\left(H e_{i}-A e_{i}\right)^{2}=0
$$

Similarly, we get

$$
\sum_{i=1}^{2 n} \beta\left(H e_{i}-A e_{i}\right)^{2}=0
$$

and we conclude

$$
\begin{aligned}
\|F(H I-A)\|^{2} & =\|H I-A\|^{2} \\
& =2 n H^{2}+\|A\|^{2}-4 n H^{2} \\
& =\|A\|^{2}-2 n H^{2} .
\end{aligned}
$$

Thus, Equation (52) gives

$$
\left\|A_{\rho}\right\|^{2}=\rho^{2}\left(H^{2}\|A\|^{2}+2 n+4 n H^{2}\right)+\left(\|A\|^{2}-2 n H^{2}\right) .
$$

Now, using Equation (50) and above Equation, we conclude

$$
\begin{aligned}
\left\|A_{\rho}\right\|^{2}-\frac{1}{2 n}(\Delta \rho)^{2}= & \rho^{2}\left(H^{2}\|A\|^{2}+2 n+4 n H^{2}\right)+\left(\|A\|^{2}-2 n H^{2}\right) \\
& -2 n\left(1+2 H^{2}+H^{4}\right) \rho^{2},
\end{aligned}
$$

that is,

$$
\left\|A_{\rho}\right\|^{2}-\frac{1}{2 n}(\Delta \rho)^{2}=\rho^{2} H^{2}\left(\|A\|^{2}-2 n H^{2}\right)+\left(\|A\|^{2}-2 n H^{2}\right) .
$$

Thus, we have

$$
\left\|A_{\rho}\right\|^{2}-\frac{1}{2 n}(\Delta \rho)^{2}=\left(1+\rho^{2} H^{2}\right)\left(\|A\|^{2}-2 n H^{2}\right) .
$$

On the other hand, from the Bochner's formula

$$
\int_{M}\left[\operatorname{Ric}(\nabla \rho, \nabla \rho)+\left\|A_{\rho}\right\|^{2}-(\Delta \rho)^{2}\right]=0,
$$

we derive immediately

$$
\int_{M}\left[\left\|A_{\rho}\right\|^{2}-\frac{1}{2 n}(\Delta \rho)^{2}\right]=\int_{M}\left[\frac{2 n-1}{2 n}(\Delta \rho)^{2}-\operatorname{Ric}(\nabla \rho, \nabla \rho)\right] .
$$


Using now (50) and (54) in above equation, we get

$$
\begin{aligned}
\int_{M}\left(1+\rho^{2} H^{2}\right)\left(\|A\|^{2}-2 n H^{2}\right)= & \int_{M} 2 n(2 n-1)\left(1+H^{2}\right)^{2} \rho^{2} \\
& -\int_{M} \operatorname{Ric}(\nabla \rho, \nabla \rho) .
\end{aligned}
$$

On the other hand, it is obvious that Equation (50) implies $\rho \Delta \rho=-2 n\left(1+H^{2}\right) \rho^{2}$ and integrating this equation by parts, we conclude

$$
\int_{M}\|\nabla \rho\|^{2}=2 n\left(1+H^{2}\right) \int_{M} \rho^{2}
$$

Using this equation in (55), we get

$$
\int_{M}\left(1+\rho^{2} H^{2}\right)\left(\|A\|^{2}-2 n H^{2}\right)=\int_{M}\left[(2 n-1)\left(1+H^{2}\right)\|\nabla \rho\|^{2}-\operatorname{Ric}(\nabla \rho, \nabla \rho)\right] .
$$

Using inequality (47) in above equation, we conclude

$$
\int_{M}\left(1+\rho^{2} H^{2}\right)\left(\|A\|^{2}-2 n H^{2}\right) \leq 0
$$

and in view of Schwartz's inequality $\|A\|^{2} \geq 2 n H^{2}$, the integrand in above inequality is non-negative. Hence, we derive

$$
\left(1+\rho^{2} H^{2}\right)\left(\|A\|^{2}-2 n H^{2}\right)=0,
$$

that is, $\|A\|^{2}-2 n H^{2}=0$, which implies the equality in the Schwartz's inequality. However, this holds if and only if $A=H I$. Thus $M$ is a totally umbilical hypersurface of constant mean curvature $H$ and this proves that $M$ is isometric to the sphere $\mathbf{S}^{2 n}\left(\frac{1}{\sqrt{1+H^{2}}}\right)$.

The converse statement follows trivially since if we suppose that $M$ is isometric to $\mathbf{S}^{2 n}\left(\frac{1}{\sqrt{1+H^{2}}}\right)$, then it is well known that the first nonzero eigenvalue $\lambda_{1}$ of the Laplace operator is given by $\lambda_{1}=2 n\left(1+H^{2}\right)$.

As an immediate consequence of Theorem 5, we have the following result for hypersurfaces of the unit sphere $\mathbf{S}^{2 n+1}$.

Corollary 2. Let $M$ be a compact and connected orientable hypersurface of constant mean curvature $H$ of the unit sphere $\mathbf{S}^{2 n+1}$ such that $A \xi^{t}=\mu \xi^{T}$ for a constant $\mu$. Then the first nonzero eigenvalue $\lambda_{1}$ of the Laplace operator on $M$ satisfies

$$
\lambda_{1} \leq 2 n\left(1+H^{2}\right) .
$$

Moreover, the equality holds if and only if $M$ is isometric to the sphere $\mathbf{S}^{2 n}\left(\frac{1}{\sqrt{1+H^{2}}}\right)$.

Remark 1. Next, we would like to point out the existence of hypersurfaces which satisfy the condition that the tangential component of the Reeb vector field to the hypersurface is a principal direction, providing two non-trivial examples.

First, we consider the unit sphere $\mathbf{S}^{2 n+1} \subset \mathbf{R}^{2 n+2}$ equipped with the standard Sasakian structure $(\varphi, \xi, \eta, g)[1]$. We shall write

$$
\mathbf{S}^{2 n+1}=\left\{\left(u_{1}, u_{2}, \ldots, u_{2 n+1}, u_{2 n+2}\right) \in \mathbf{R}^{2 n+2}:\|u\|=1\right\}
$$


and let us denote by $J$ the canonical complex structure on $\mathbf{R}^{2 n+2}$ defined as

$$
J\left(u_{1}, u_{2}, u_{3}, u_{4}, \ldots, u_{2 n+1}, u_{2 n+2}\right)=\left(-u_{2}, u_{1},-u_{4}, u_{3}, \ldots,-u_{2 n+2}, u_{2 n+1}\right)
$$

and by $\bar{N}=\left(u_{1}, \ldots, u_{2 n+2}\right)$ the unit normal vector field to $\mathbf{S}^{2 n+1}$. Then it is known that the Reeb vector field on $\mathbf{S}^{2 n+1}$ is given by $\xi=-J \bar{N}$, that is,

$$
\xi=\left(u_{2},-u_{1}, \ldots, u_{2 n+2},-u_{2 n+1}\right),
$$

$\eta$ is the 1-form dual to $\xi$ with respect to the induced metric $g$ (by the Euclidean metric on $\mathbf{R}^{2 n+2}$ ), while $\varphi(X)=J X-\eta(X) \bar{N}, X \in \mathcal{X}\left(\mathbf{S}^{2 n+1}\right)$. Now, consider

$$
M=\left\{\left(u_{1}, u_{2}, \ldots, u_{2 n+1}, u_{2 n+2}\right) \in \mathbf{R}^{2 n+2}: \sum_{i=1}^{2 n+1} u_{i}^{2}=c^{2}, u_{2 n+2}=\sqrt{1-c^{2}}, 0<c<1\right\}
$$

Then $M$ is a hypersurface of $\mathbf{S}^{2 n+1}$ with unit normal

$$
N=\left(-\frac{\sqrt{1-c^{2}}}{c} u_{1}, \ldots,-\frac{\sqrt{1-c^{2}}}{c} u_{2 n+1}, c\right)
$$

Therefore, we have

$$
\sigma=g(\xi, N)=-\frac{1}{c} u_{2 n+1}
$$

and it gives

$$
\xi^{T}=\xi-\sigma N=\xi+\frac{1}{c} u_{2 n+1} N
$$

that is,

$$
\xi^{T}=\left(u_{2}-\frac{\sqrt{1-c^{2}}}{c^{2}} u_{1} u_{2 n+1},-u_{1}-\frac{\sqrt{1-c^{2}}}{c^{2}} u_{2} u_{2 n+1}, \ldots, u_{2 n+2}-\frac{\sqrt{1-c^{2}}}{c^{2}} u_{2 n+1}^{2}, 0\right) .
$$

Let $A$ be the shape operator and $\bar{\nabla}$ be the Riemannian connection on $\mathbf{S}^{2 n+1}$ and $D$ be the Euclidean connection on $\mathbf{R}^{2 n+2}$. Then using (56), we have

$$
-A \xi^{T}=\bar{\nabla}_{\xi^{T}} N=D_{\xi^{T}} N=\left(-\frac{\sqrt{1-c^{2}}}{c} \xi^{T}\left(u_{1}\right), \ldots,-\frac{\sqrt{1-c^{2}}}{c} \xi^{T}\left(u_{2 n+1}\right), 0\right) .
$$

which on using (57), gives

$$
A \xi^{T}=\frac{\sqrt{1-c^{2}}}{c} \xi^{T}
$$

Hence, $\xi^{T}$ is principal direction with constant principal curvature $\frac{\sqrt{1-c^{2}}}{c}>0$ and we derive that $M$ provides us a first example of hypersurface satisfying the condition that the tangential component of the Reeb vector field to the hypersurface is a principal direction.

Similarly, one can show that the hypersurface $\mathbf{S}^{2 n}$ of the Sasakian manifold $\mathbf{R}^{2 n+1}(\varphi, \xi, \eta, g)$ gives another example of hypersurface with such a property. Recall that if $\left(x_{i}, y_{i}, z\right), i=1, \ldots, n$, are the coordinates on $\mathbf{R}^{2 n+1}$, then the Sasakian structure $(\varphi, \xi, \eta, g)$ on $\mathbf{R}^{2 n+1}$ is given by [1]

$$
\varphi=\left(\begin{array}{ccc}
0 & \delta_{i j} & 0 \\
-\delta_{i j} & 0 & 0 \\
0 & y^{j} & 0
\end{array}\right), \xi=2 \frac{\partial}{\partial z}, \eta=\frac{1}{2}\left(d z-\sum_{i=1}^{n} y^{i} d x^{i}\right), g=\eta \otimes \eta+\frac{1}{4} \sum_{i=1}^{n}\left[\left(d x^{i}\right)^{2}+\left(d y^{i}\right)^{2}\right]^{2} .
$$


Author Contributions: Conceptualization and methodology, H.A., S.D. and N.B.T.; formal analysis, G.-E.V.; writing original draft preparation, H.A. and G.-E.V.; writing-review and editing, S.D. and G.-E.V.; supervision, S.D. and G.-E.V.; project administration, H.A. and N.B.T.; and funding acquisition, N.B.T. All authors have read and agreed to the published version of the manuscript.

Funding: The authors extend their appreciations to the Deanship of Scientific Research King Saud University for funding this work through research group no (RG-1440-142).

Conflicts of Interest: The authors declare no conflicts of interest.

\section{References}

1. Blair, D.E. Riemannian Geometry of Contact and Symplectic Manifolds, 2nd ed.; Birkhäuser: Boston, MA, USA, 2010.

2. Adachi T.; Kameda M.; Maeda, S. Geometric meaning of Sasakian space forms from the view point of submanifold theory. Kodai Math. J. 2010, 33, 383-397. [CrossRef]

3. Bejancu A.; Deshmukh, S. Real hypersurfaces of $C P^{n}$ with non-negative Ricci curvature. Proc. Am. Math. Soc. 1996, 124, 269-274. [CrossRef]

4. Deshmukh, S. Real hypersurfaces in a Euclidean complex space form. Quart. J. Math. 2007, 58, $303-307$. [CrossRef]

5. Deshmukh, S. Real hypersurfaces of a complex space form. Monatsh. Math. 2012, 166, 93-106. [CrossRef]

6. Kaimakamis, G.; Panagiotidou, K.; Pérez, J.D. Derivatives of the operator $\phi A-A \phi$ on a real hypersurface in non-flat complex space forms. Bull. Malays. Math. Sci. Soc. 2020, 43, 267-282. [CrossRef]

7. Kimura, M.; Maeda, S. On real hypersurface of a complex projective space. Math. Z. 1989, 202, $299-312$. [CrossRef]

8. Maeda, S.; Tanabe, H.; Udagawa, S. Generating curves of minimal ruled real hypersurfaces in a nonflat complex space form. Canad. Math. Bull. 2019, 62, 383-392. [CrossRef]

9. Niebergall, R.; Ryan, P.J. Real hypersurfaces in complex space forms. In Tight and Taut Submanifolds; Cecil, T.E., Chern, S.S., Eds.; Cambridge University Press: New York, NY, USA, 1998; pp. 233-305.

10. Sasahara, T. Ricci curvature of real hypersurfaces in non-flat complex space forms. Mediterr. J. Math. 2018, 15, 12. [CrossRef]

11. Wang, Y. Cyclic $\eta$-parallel shape and Ricci operators on real hypersurfaces in two-dimensional nonflat complex space forms. Pacific J. Math. 2019, 302, 335-352. [CrossRef]

12. Boyer C.; Galicki, K. Sasakian Geometry; Oxford Mathematical Monographs; Oxford University Press: Oxford, NY, USA, 2008.

13. Slesar, V.; Visinescu, M.; Vîlcu, G.E. Toric data, Killing forms and complete integrability of geodesics in Sasaki-Einstein spaces $Y^{p, q}$. Ann. Phys. 2015, 361, 548-562. [CrossRef]

14. Watanabe, Y. Totally umbilical surfaces in normal contact Riemannian manifold. Kodai Math. Sem. Rep. 1967, 19, 474-487. [CrossRef]

15. Obata, M. Conformal transformations of Riemannian manifolds. J. Diff. Geom. 1970, 4, 311-333. [CrossRef]

16. Obata, M. The conjectures about conformal transformations. J. Diff. Geom. 1971, 6, 247-258. [CrossRef]

17. Yamaguchi, S. On hypersurfaces in Sasakian manifolds. Kodai Math. Sem. Rep. 1969, 21, 64-72. [CrossRef]

18. Chen B.-Y. Pseudo-Riemannian Geometry, $\delta$-Invariants and Applications; World Scientific: Hackensack, NJ, USA, 2011.

19. Besse, A.L. Einstein Manifolds; Springer: Berlin/Heidelberg, Germany, 1987. 